\title{
BMJ Open Efficacy and safety of ginger-salt- indirect moxibustion for urge urinary incontinence after stroke: protocol for a pilot multicentre randomised controlled trial
}

\author{
Linpeng Wang, ${ }^{1}$ Lichen Wang, ${ }^{1}$ Guangxia Shi, ${ }^{1}$ Lin Zeng, ${ }^{2}$ Yi Yang, ${ }^{1}$ Tao Zhang, ${ }^{1}$ \\ Huilin Liu ${ }^{1}$
}

To cite: Wang $L$, Wang $L$, Shi $\mathrm{G}$, et al. Efficacy and safety of ginger-salt-indirect moxibustion for urge urinary incontinence after stroke: protocol for a pilot multicentre randomised controlled trial. BMJ Open 2014:4:e006326. doi:10.1136/bmjopen-2014006326

- Prepublication history for this paper is available online. To view these files please visit the journal online (http://dx.doi.org/10.1136/ bmjopen-2014-006326).

Received 7 August 2014 Revised 19 September 2014 Accepted 24 September 2014

CrossMark

\footnotetext{
${ }^{1}$ Acupuncture and Moxibustion Department, Beijing Hospital of Traditional Chinese Medicine affiliated to Capital Medical University, Beijing, China

${ }^{2}$ Research Center of Clinical Epidemiology Affiliated to Peking University, Beijing, China
}

Correspondence to Dr Huilin Liu; Ihlxwy@aliyun.com

\section{ABSTRACT}

Introduction: Ginger-salt-indirect moxibustion is widely applied to treat urge urinary incontinence after stroke, which is a common complication in stroke survivors. Moxa cone moxibustion and moxa box moxibustion are the main techniques of ginger-saltindirect moxibustion. Our previous study had shown that ginger-salt-indirect moxibustion using moxa cones was feasible and effective for urination disorders poststroke. This pilot study aims to assess the feasibility of conducting research to evaluate the efficacy and safety of ginger-salt-indirect moxibustion for patients with post-stroke urge urinary incontinence.

Methods and analysis: This is a multicentre, prospective, single-blinded, pilot randomised controlled trial. 120 eligible patients will be randomly allocated to three groups. Treatment group A $(n=40)$ will receive moxa cone moxibustion and routine care; treatment group $B(n=40)$ will receive moxa box moxibustion and routine care; control group $(n=40)$ will only receive routine care for stroke recovery. The entire moxibustion treatment will consist of a total of 28 sessions during the course of 4 weeks. The primary outcome measure will be the increase in mean volume per void assessed at week 4 from the first moxibustion session (baseline). Secondary outcome measures will include mean frequency of urination per day and quality of life assessments measured by completion of the Incontinence Quality of Life Questionnaire and Barthel Index. All outcome measures will be assessed at baseline and at 4 and 16 weeks from baseline. Adverse events in the three groups will be recorded to assess the safety of moxibustion.

Ethics and dissemination: Research ethics was approved by the Research Ethical Committee of Beijing Hospital of Traditional Chinese Medicine Affiliated to the Capital Medical University (ref: 2013BL-094). Written informed consent will be obtained from all participants. Study results will be published in peer reviewed journals.

Trial registration number: ISRCTN 44706974.

\section{Strengths and limitations of this study}

First pilot study to evaluate the feasibility of conducting research to assess the efficacy and safety of ginger-salt-indirect moxibustion as treatment for urge urinary incontinence after stroke.

- Multicentre, randomised controlled trial with pragmatic design.

- Interventions conducted by certified acupuncturists according to the STRICTOM and good clinical practice guidelines (GCP).

- Lack of blinding of acupuncturists and participants due to the nature of moxibustion.

\section{INTRODUCTION}

Urinary incontinence (UI) is defined by the International Continence Society (ICS) as involuntary leakage of urine that causes hygienic or social problems. ${ }^{1}$ The prevalence of UI after stroke varies from $32-79 \%$ at admission, ${ }^{2-4} 25 \%$ at hospital discharge, and $15 \%$ after 1 year. ${ }^{4}$ Urge urinary incontinence (UUI) is a common type of $\mathrm{UI}^{5}$ that occurs after stroke. It is characterised by involuntary leakage of urine accompanied or preceded by urgency. ${ }^{1}$

The results of a previous review ${ }^{6}$ suggested that there were three major causes of UI after stroke: detrusor hyperreflexia caused by infarction; stroke-related cognitive or language deficits; and bladder hyporeflexia with resultant overflow incontinence. UUI can be caused by detrusor overactivity due to the loss of inhibitory neurones after stroke. ${ }^{7}$ Detrusor overactivity is characterised by involuntary detrusor contractions during the filling and storage phase. ${ }^{1} 78$ When intradetrusor pressure generated by abnormal 
detrusor contractions exceeds sphincter pressure, UI occurs. ${ }^{7}$ UI is a strong prognostic indicator for stroke recovery, which is associated with high rates of mortality, ${ }^{9}{ }^{10}$ disability $^{11}$ and increasing admission to institutional care. ${ }^{12}$ Micturition disorders may result in urinary infection, nephritis, fungal dermatitis, ${ }^{13}$ and even bedsores. Typical symptoms, including frequent micturition, nocturia and urgency, could lead to impaired quality of life $^{14}$ and heavy economic burden. ${ }^{15} 16$

Initial treatments for UUI after stroke recommended by ICS are behavioural therapy and pharmacotherapy. ${ }^{1}$ Behavioural therapies, including healthy bladder habits and training techniques, are aimed at changing patient lifestyle and at teaching patients to control urgency and enhance continence ability. ${ }^{17}$ For habit modification, patients are given guidance on how to re-establish a healthy voiding schedule, eliminate bladder irritants from the diet, manage fluid intake and bowel regularity, control weight, and give up smoking. ${ }^{18-24}$ Training techniques consist of bladder training, urgency control techniques, pelvic muscle exercises, delayed voiding, and multicomponent behavioural training. ${ }^{17}$ Behavioural interventions are applied in the primary care setting, with or without pharmacotherapy. ${ }^{25-27}$ Among these strategies, behavioural training and bladder training have the strongest evidence of efficacy for the treatment of UUI. ${ }^{28-31}$

Antimuscarinic drugs can reduce urgency and improve bladder function by controlling detrusor muscle overactivity through inhibition of M2 and M3 muscarinic bladder receptors. ${ }^{32-34}$ Several meta-analyses have shown that the most widely used antimuscarinic drugs have significant clinical benefits on UUI. ${ }^{35-38}$ However, use of antimuscarinic agents is complicated by dose convenience, drug contraindication, and financial concerns. ${ }^{39}$ A Cochrane systematic review ${ }^{40}$ concluded that no studies designed to manage UI after stroke in secondary care had been rigorously conducted so far. Although there are clinical guidelines for the management of UI after stroke, ${ }^{1}$ to date there is insufficient good quality evidence to support current clinical practices. ${ }^{41}$ More available therapies and well-designed studies are required to provide further evidence for management of UUI after stroke.

Moxibustion is a therapy that uses ignited materials (usually moxa) to heat selected points of the skin surface. This therapy is widely used for chronic deficiency diseases ${ }^{42}$ and is commonly indicated for UUI. ${ }^{43}$ The mechanism of action of moxibustion combines thermal, radiation and pharmacological effects of the materials used, acting on the meridian system. ${ }^{44}$ Following the theory of Traditional Chinese Medicine (TCM), kidney $q i$ regulates voiding function and ensures voluntary urination. Deficiency of kidney $q i$ is known to be the primary pathogenesis of UI according to 'ZhuBingYuanHouLun' (a famous ancient TCM literature). Ginger and salt are typically used on Shenque (CV8) acupoint to warm, tonify and nourish the kidney $q i$, thereby controlling the bladder and regulating voiding function.

Moxa cone moxibustion and moxa box moxibustion are primary techniques of ginger-salt-indirect moxibustion, which are commonly used in clinical practices in China. Moxa cone moxibustion involves a moxa cone burning directly on the ginger slice and providing thermal stimulus to the skin surface. Moxa box moxibustion facilitates manipulations and reduces adverse reactions by using the moxa box device with moxa sticks inside over the ginger slice. Results of our previous study ${ }^{45}$ showed that ginger-salt-partitioned moxibustion with moxa cone could reduce mean daytime and nighttime voiding frequency in the treatment of urination disorders post-stroke. In addition, we also observed that ginger-salt-indirect moxibustion with moxa cones could increase mean volume per void of UI after stroke in an unpublished study. Our previous studies, together with results from an earlier Cochrane review, ${ }^{40}{ }^{45}$ suggest ginger-salt-indirect moxibustion may be worth investigating with more rigorous study design.

\section{METHODS}

\section{Objectives}

This pilot study aims to evaluate the feasibility of research to assess the efficacy and safety of ginger-salt-indirect moxibustion for the treatment of UUI after stroke.

\section{Recruitment}

This is a multicentre, single-blinded, pilot randomised controlled trial. The research consists of three sequential parts (figure 1): a recruitment period before randomisation, a treatment period of 4 weeks, and a follow-up period of 12 weeks. One hundred and twenty eligible participants will be recruited from acupuncture wards of three hospitals according to the inclusion and exclusion criteria. During the first visit, potentially qualified patients will be provided with detailed information about this study, including the research objective, study procedure, potential benefits and risks. If a patient shows willingness to participate, they will be required to voluntarily sign a written informed consent. This will be followed by baseline assessment and randomisation.

\section{Design}

\section{Randomisation and allocation concealment}

The randomisation scheme is provided by the Research Center of Clinical Epidemiology Affiliated to Peking University Third Hospital in China. Statistical experts will use the block randomisation method (block size of 6) of the SAS package (V.9.1.3; SAS institute Inc, Cary, North Carolina, USA) to form the random allocation sequence. Then computer-generated opaque sealed envelopes, each with the serial number outside and group number inside, will be produced. The envelopes will be kept in a secure locked drawer making it inaccessible to all study personnel. After baseline assessments, 
Figure 1 Trial flowchart.

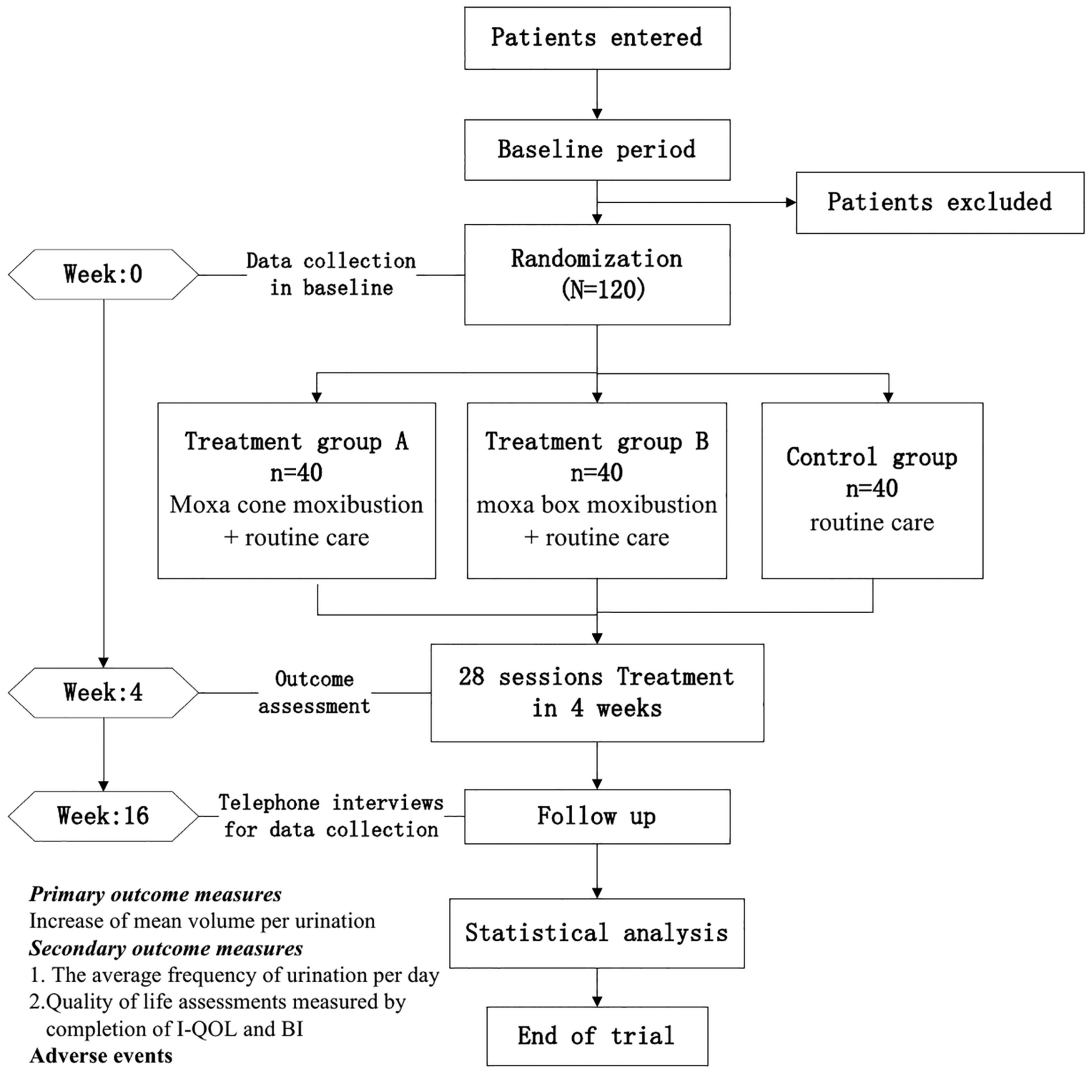

the envelopes will be opened sequentially by an independent researcher in front of the participants to ensure adequate concealment. Participants will be allocated to three groups according to the group number printed inside the envelopes.

\section{Blinding}

Due to the nature of moxibustion, it is difficult to ensure the therapists or participants are blinded to the treatment allocation. Data managers and statisticians will be blinded throughout the trial. During the intervention, therapists and data managers will be requested not to communicate with each other about the allocation. Blinded telephone interviewers will collect the follow-up materials to evaluate the long term effect of moxibustion at 16 weeks after the baseline.

\section{Participants}

\section{Sample size}

Because this is a pilot study, a power calculation to determine the sample size was not conducted. Sample size calculation was performed based on estimates of the number of participants we would expect to recruit within 24 months. We therefore plan to recruit five participants per month, according to our previous trial. A sample size of 40 per group and a total number of 120 will be included, which is larger than the minimum of 12 per group suggested for pilot studies. ${ }^{46}$ Outcomes of this pilot study will help calculate the appropriate sample size for further randomised clinical trials.

\section{Inclusion criteria}

1. Male or female, aged 40-75 years

2. Inpatients with UUI after stroke, according to the diagnosis criteria of the American Stroke Association and the ICS

3. 4 th to 48 th week after stroke onset

4. Normal consciousness, communication ability and recognition

5. Written informed consent.

\section{Exclusion criteria}

1. UUI caused by spinal injury, multiple sclerosis or hyperplasia of the prostate gland

2. Chronic urinary retention or UI before stroke onset

3. Stress UI, mixed UI and chronic urinary tract infection

4. Insufficiency of heart, liver, kidney organs

5. Participants in another clinical trial.

\section{Discontinuation criteria}

Reasons for discontinuation of treatment may include, but are not limited to, the following:

1. Participant's decision to discontinue treatment at any time for any reason

2. Investigator's determination to discontinue treatment for the patient's safety and best interests at any time

3. Non-compliance of participants with the study procedure (eg, study visits)

4. Concomitant therapy that could affect the study results during the trial

5. Detection of protocol violations at any time. 


\section{Intervention}

Ginger-salt-indirect moxibustion is formulated on the basis of descriptions in the ancient literature and our clinical experience. Moxibustion will be manipulated by certified acupuncturists with at least 20 years' clinical experience. All treatment details will be standardised between practitioners by guiding videos and relative training before the first acupuncture session. Interventions will be performed in accordance with the STRICTOM $^{47}$ and good clinical practice guidelines (GCP).

\section{Treatment group A}

Participants in treatment group A will receive moxa cone moxibustion and routine care once a day for 4 weeks. The procedure for each session as it is used in normal practices is as follows (figure 2). Participants are asked to lie on a bed in a supine position and remove clothing to fully expose the navel in a temperature controlled room $\left(25-30^{\circ} \mathrm{C}\right)$. A certain amount of salt is put on the navel to cover Shenque (CV8) acupoint and is covered by a fresh slice of ginger $(30 \mathrm{~mm}$ in diameter and 4-5 $\mathrm{mm}$ in thickness). Then a moxa cone (pure wormwood fibre in material, $15 \mathrm{~mm}$ in diameter and $30 \mathrm{~mm}$ in length; Tongrentang Inc, China) is placed on the fresh ginger slice and lit by the therapist. Once the moxa cone is burnt out, the therapist removes the whole moxa cone and replaces it with another one. The technique requires the participant to experience a sensation of heat but no painful burning sensation. If participants feel pain or a burning sensation, the ginger slice with burning moxa cone will be removed immediately and reset after several minutes. Each session requires three units of moxa cone. The therapist is required to observe the patient carefully and quickly remove the burning ash to avoid injury. Because this study is designed to evaluate moxibustion treatment as it is used in normal practice, the moxibustion group will also receive the usual care provided to the control group.

\section{Treatment group B}

Participants in treatment group B will receive moxa box moxibustion and routine care once a day for 4 weeks. The manipulations are generally similar to those in treatment group A. The only difference is that the doublehole moxa box $(13 \times 8 \times 8.5 \mathrm{~cm})$ with two moxa sticks (pure wormwood fibre, $15 \mathrm{~mm}$ in diameter and $70 \mathrm{~mm}$ in length; Tongrentang Inc, China) in the holes will be placed on the fresh ginger slice (figure 3). The moxa sticks will be ignited from the bottom. If patients feel pain, the therapist will remove the lid with the moxa sticks for several minutes. Each session requires three units of moxa sticks.

\section{Control group}

According to pragmatic design, the control group will receive routine therapies for stroke recovery. These include control of blood pressure, inhibition of platelet aggregation, routine physiotherapy, occupational therapy, and glucose control treatment. It will be suggested to the participants in this group that they maintain their normal lifestyle, including diet, exercise and workload. Moreover, participants will be encouraged to inform the researchers of any new treatments performed after entry into the trial.

\section{Outcome measures}

\section{Primary outcome measure}

The primary outcome measure will be the increase in mean volume per void at week 4 from the baseline.

Mean volume per void is one of the main quantitative indicators recorded in bladder diaries or frequency volume charts. ${ }^{17} 31{ }^{48-50}$ It is calculated by dividing the total volume of voluntary urination by the total frequency of voluntary urination, based on $72 \mathrm{~h}$ frequencyvolume chart data. The increase in mean volume per void objectively reflects an improvement in bladder capacity and detrusor stability in the urine filling and storage period. In a previous study, we observed that mean volume per void increased in the ginger-salt-indirect moxibustion group compared to the control group, the difference being statistically significant. Consequently, we selected the increase in mean volume per void at week 4 from baseline as the primary outcome. We will also assess the increase in mean volume per void at week 16 from baseline to evaluate long term efficacy.

\section{Secondary outcome measures}

1. The mean frequency of urination per day, including voluntary and incontinence urination.

2. Quality of life assessments measured by completion of the Incontinence Quality of Life Questionnaire $(\mathrm{I}-\mathrm{QOL})^{51}$ and the Barthel Index (BI) ${ }^{52}$

The average frequency of urination per day is composed of mean frequency of voluntary and incontinent urination, which represents the severity of frequent micturition and UI, respectively. This is strongly linked to the stability of the bladder and detrusor. Questionnaires for quality of life assessment will be completed based on the objective condition and subjective sensation of the participants.

All outcome measures will be assessed before the baseline, and at 4 and 16 weeks from the baseline.

\section{Adverse events}

Adverse events (AEs) are defined as negative or unintended clinical manifestations following the treatment. Participants will be instructed to report any abnormal reactions to the clinical research team at any time. In addition, study investigators will collect information about abnormal reactions weekly by visiting the participants. All details of related and unexpected AEs, such as time of occurrence, degree of $\mathrm{AE}$, and suspected causes, will be recorded on Case Report Forms (CRFs). The common AEs related to moxibustion include allergy, 


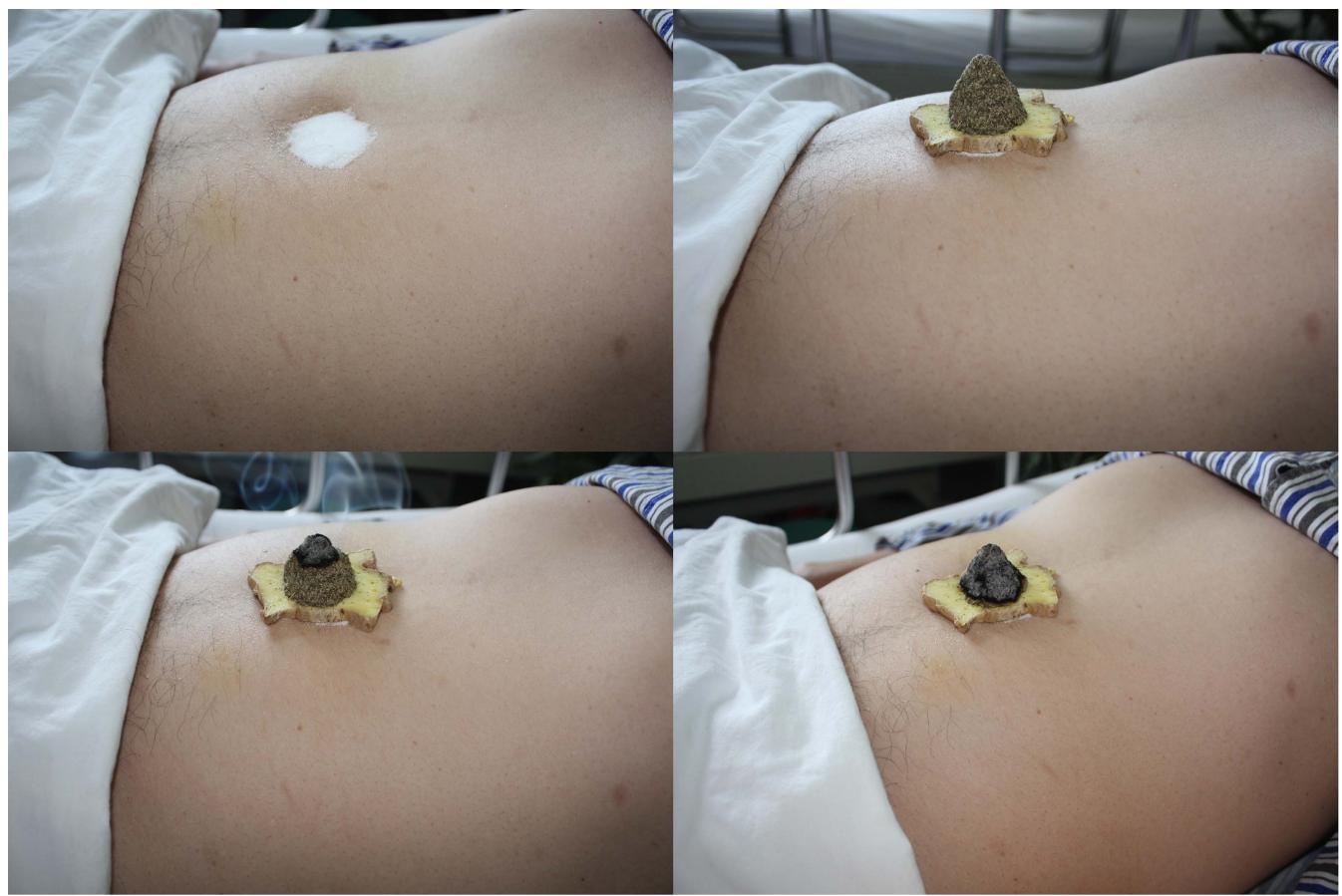

Figure 2 Moxa cone moxibustion.

burn and infection. ${ }^{53}$ AEs will be classified into five grades: mild (asymptomatic or mild symptoms; clinical or diagnostic observations only; intervention not indicated); moderate (minimal, local or non-invasive intervention indicated); severe (severe or medically significant but not immediately life-threatening; hospitalisation or prolongation of hospitalisation indicated); very severe (life-threatening consequences; urgent intervention indicated); death related to AEs. Participants with mild and moderate AEs will receive symptomatic treatment and will be closely followed up by the research team. Severe AEs will be reported to the Research Ethics Committee within $48 \mathrm{~h}$. The Research Ethics Committee will offer medical advice to the research team and determine whether the patient is eligible for further treatment.

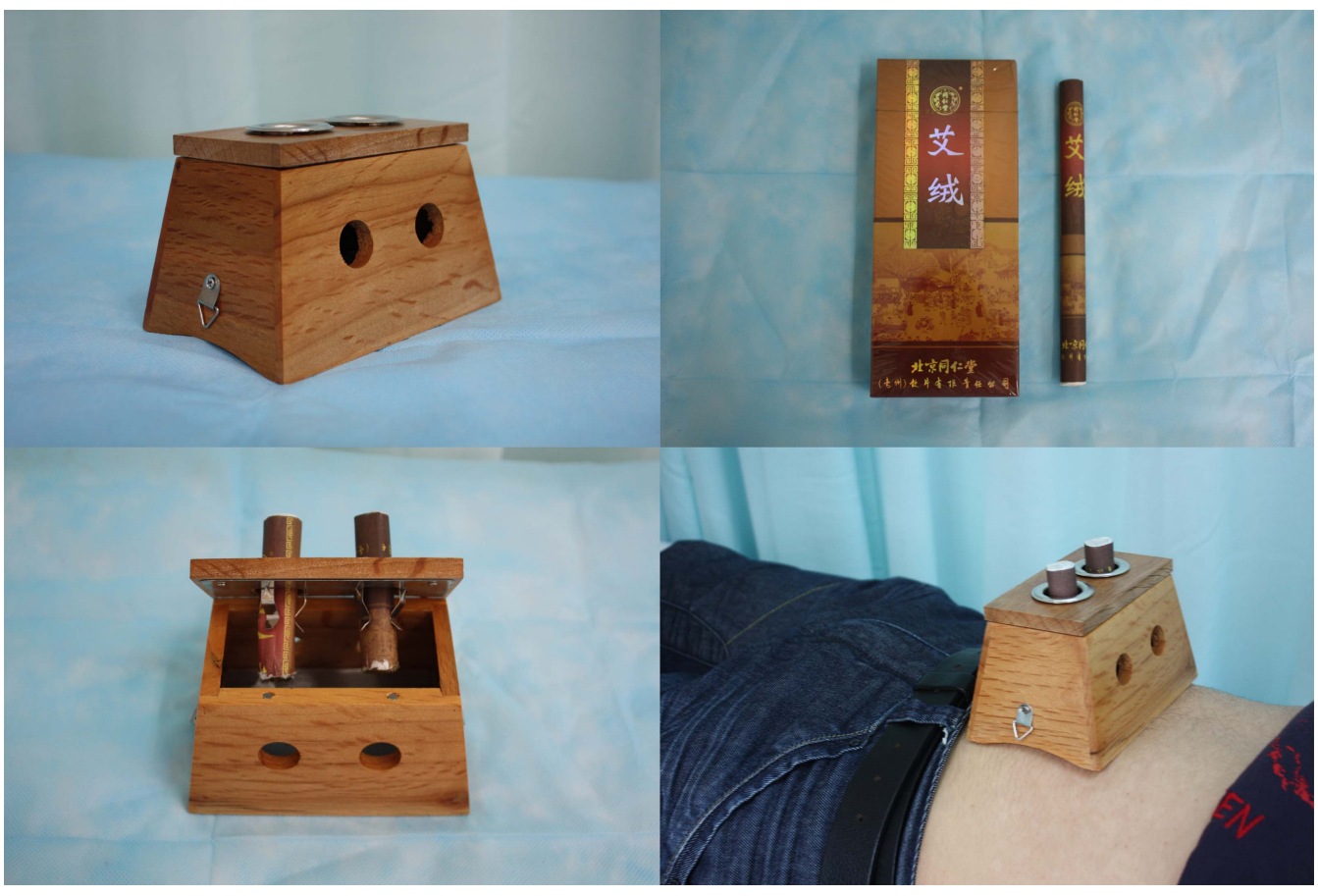

Figure 3 Moxa box moxibustion. 


\section{Data management}

Before recruitment, the whole research group, including therapists, data administrators and outcome assessors, will participate in a training seminar about research contents and data management. Baseline characteristics of participants will be recorded on CRFs by a research assistant. Data collection of urination and questionnaires will be conducted by a researcher who remains blinded to the group allocation at the baseline, 4 and 16 weeks from the baseline. Occupational caregivers will be trained to identify UI and voluntary urination. Caregivers will collect urine following voluntary urination and measure it with a pot-shaped urine collector with scales. They will be trained to record accurate voiding time, voluntary urinary volume and UI episodes on a $72 \mathrm{~h}$ frequency-volume chart (figure 4). The record will be started at 8:00 and last for $72 \mathrm{~h}$. If caregivers failed to collect urine in time, they will note voiding time and specific reasons on the chart. The blinded researcher will complete the urination section on CRFs according to the $72 \mathrm{~h}$ frequency-volume charts handed in by caregivers.

Two independent researchers blinded to the group allocation will enter the data on an Excel spreadsheet after the completion of the CRFs separately. Another independent researcher will compare the two datasets for check-up. If different data entry is discovered, data will be compared with the original CRFs to verify inconsistency. All modification will be marked on the CRFs. Research data will be gathered and saved, abiding by the Data Protection Act 1998. Paper files will be kept in a locked filing cabinet. Electronic documents will be stored in a password protected computer, with access restricted to the principal investigator. All research documents will be preserved for at least 5 years after publication.

\section{Statistical analysis}

Data analysis will be performed in a blinded pattern by statisticians of the Research Center of Clinical Epidemiology Affiliated to Peking University Third Hospital in China. Statistical analyses will be conducted on an intention-to-treat basis using the SPSS statistical package programme (V.18.0). Missing data will be replaced in

72-hour frequency-volume chart

\begin{tabular}{|c|c|c|c|c|}
\hline Date & $\begin{array}{c}\text { Time } \\
\text { (a.m/p.m) }\end{array}$ & $\begin{array}{c}\text { Volume voided } \\
(\mathbf{m l})\end{array}$ & $\begin{array}{c}\text { urinary incontinence } \\
\text { (tick in the blank) }\end{array}$ & notes \\
\hline & & & & \\
\hline & & & & \\
\hline & & & & \\
\hline & & & & \\
\hline & & & & \\
\hline & & & & \\
\hline
\end{tabular}

Figure $472 \mathrm{~h}$ frequency-volume chart. accordance with the principle of the last observation carried forward. A value of $\mathrm{p}<0.05$ will be considered statistically significant with a two-sided test. Baseline characteristics including gender, age and previous duration are described as n (\%) for categorical data and mean \pm SD for continuous data. To compare the differences among groups, we will perform analysis of variance for normally distributed data, the Kruskal-Wallis test for abnormally distributed data, and the $\chi^{2}$ test for categorical data. Comparisons between two groups will be conducted using the Bonferroni method of post hoc multiple comparisons.

\section{Ethical considerations}

This study adheres to the principles of the Declaration of Helsinki and has been approved by the Research Ethical Committee of Beijing Hospital of TCM Affiliated to Capital Medical University (ref: 2013BL-094). We will conduct the research in the following hospitals: Beijing TCM Hospital Affiliated to Capital Medical University, Beijing Huguosi Hospital of TCM and China Rehabilitation Research Center. Each participant will sign written informed consents voluntarily.

\section{DISCUSSION}

Placebo or sham control is encouraged in clinical trials to avoid bias. ${ }^{54}$ To our knowledge, no consensus has been established to recommend valid placebo or sham methods of moxibustion so far. Sham moxibustion is impractical for blinding purposes because of the common knowledge of moxibustion among Chinese patients. Owing to the preference for moxibustion, non-compliance of Chinese patients will make it difficult to use standard therapies as control treatment. For pragmatic purposes, we set a usual care group as a blank control group, rather than a certain effective therapy or a sham device.

In regard to the evaluation instruments, we selected a $72 \mathrm{~h}$ frequency-volume chart to record urination details, and I-QOL together with BI to assess quality of life. The frequency-volume chart and bladder diary is highly recommended in clinical trials by ICS. ${ }^{1}$ Frequencyvolume charts record the time and volume of each voiding, as well as the incontinence episodes. ICS suggested that frequency-volume charts should be recorded for at least $24 \mathrm{~h}^{1}{ }^{1}$ A minimum of $72 \mathrm{~h}$ was required to ensure reliability for diary parameters. ${ }^{48}{ }^{55-58}$ Thus, we will use a $72 \mathrm{~h}$ frequency-volume chart to alleviate burdens for patients and improve compliance. ${ }^{59} 60$

As for the assessment of quality of life, I-QOL is strongly recommended to assess the effect of UI on patient quality of life. ${ }^{61}$ Previous research ${ }^{51}$ has affirmed the reliability and validity of $\mathrm{I}-\mathrm{QOL}$ as an incontinence-related $\mathrm{QOL}$ instrument in neurogenic UI patients. The items of I-QOL focus on three dimensions, namely social embarrassment, avoidance behaviour, and psychosocial impact. BI is added to evaluate patients' activities of daily living, which is recognised as a valid and reliable outcome measurement for stroke survivors. $^{52}$ 
One limitation of this pilot study is that we will be unable to prevent therapists and participants from knowing the group allocation because of the characteristics of moxibustion. To minimise the bias, therapists will receive professional training and strict quality control. In order to homogenise the psychological effects, participants are informed that the effects of the two moxibustion techniques are uncertain. The words 'placebo' or 'control' will be avoided. Similar strategies have been applied in previous trials. ${ }^{62}{ }^{63}$ Non-compliance of the control group is another limitation of this pilot trial. It may lead to a high dropout rate. Participants will be provided with 150 Renminbi (RMB) as financial compensation to improve compliance.

This study is the major sub-project focusing on stroke recovery, which is sponsored and funded by the National Administration of TCM of China. This protocol describes the first pilot randomised controlled trial evaluating the feasibility of conducting research to assess the efficacy and safety of ginger-salt-indirect moxibustion on UUI after stroke. Results of this preliminary study will provide essential information for the design of subsequent large scale trials. Further trials will focus on the assessment of urodynamic parameters and evaluation of efficacy and safety of moxibustion on UUI after stroke.

Acknowledgements We acknowledge State Administration of Traditional Chinese Medicine of China for agreeing to sponsor this study. The authors also would like to thank Ines Janowicz for the English language editing, and the reviewers for their helpful comments on this manuscript.

Contributors LPW and HLL conceived this study and prepared the initial protocol. LW drafted the manuscript and participated in the design of the study. GXS, YY, LZ, TZ participated in revising the protocol. LZ plans for the statistical analysis. LPW and HLL made amendments to the trial protocol. All authors read and approved the final manuscript.

Funding The trial is sponsored and funded by special project for the national clinical research bases construction of traditional Chinese medicine belonging to the State Administration of Traditional Chinese Medicine of the People's Republic of China, grant number: JD2X2012152.

Competing interests None.

Patient consent Obtained.

Ethics approval Research ethics approval was attained from the Research Ethical Committee of Beijing Hospital of Traditional Chinese Medicine Affiliated to Capital Medical University (ref: 2013BL-094).

Provenance and peer review Not commissioned; externally peer reviewed.

Data sharing statement The results of this pilot study will be disseminated via peer reviewed publications and conference presentations. All of the data are available.

Open Access This is an Open Access article distributed in accordance with the Creative Commons Attribution Non Commercial (CC BY-NC 4.0) license, which permits others to distribute, remix, adapt, build upon this work noncommercially, and license their derivative works on different terms, provided the original work is properly cited and the use is non-commercial. See: http:// creativecommons.org/licenses/by-nc/4.0/

\section{REFERENCES}

1. Abrams $\mathrm{P}$, Andersson KE, Birder L, et al. Fourth International Consultation on Incontinence Recommendations of the International Scientific Committee: evaluation and treatment of urinary incontinence, pelvic organ prolapse, and fecal incontinence. Neurourol Urodyn 2010;29:213-40.
2. Brittain KR, Peet SM, Potter JF, et al. Prevalence and management of urinary incontinence in stroke survivors. Age Ageing 1999;28:509-11.

3. Marinkovic SP, Badlani G. Voiding and sexual dysfunction after cerebrovascular accidents. J Urol 2001;165:359-70.

4. Booth J, Kumlien S, Zang Y, et al. Rehabilitation nurses practices in relation to urinary incontinence following stroke: a cross-cultural comparison. J Clin Nurs 2009;18:1049-58.

5. Williams MP, Srikanth V, Bird M, et al. Urinary symptoms and natural history of urinary continence after first-ever stroke-a longitudinal population-based study. Age Ageing 2012;41:371-6.

6. Gelber DA, Good DC, Laven LJ, et al. Causes of urinary incontinence after acute hemispheric stroke. Stroke 1993;24:378-82.

7. Santiagu SK, Arianayagam M, Wang A, et al. Urinary incontinence-pathophysiology and management outline. Aust Fam Physician 2008;37:106-10.

8. Manack A, Motsko SP, Haag-Molkenteller C, et al. Epidemiology and healthcare utilization of neurogenic bladder patients in a US claims database. Neurourol Urodyn 2011;30:395-401.

9. Nakayama H, Jorgensen HS, Pedersen PM, et al. Prevalence and risk factors of incontinence after stroke. The Copenhagen Stroke Study. Stroke 1997;28:58-62.

10. Anderson CS, Jamrozik KD, Broadhurst RJ, et al. Predicting survival for 1 year among different subtypes of stroke. Results from the Perth Community Stroke Study. Stroke 1994;25:1935-44.

11. Patel M, Coshall C, Rudd AG, et al. Natural history and effects on 2 -year outcomes of urinary incontinence after stroke. Stroke 2001;32:122-7.

12. Kolominsky-Rabas PL, Hilz MJ, Neundoerfer B, et al. Impact of urinary incontinence after stroke: results from a prospective population-based stroke register. Neurourol Urodyn 2003;22:322-7.

13. Dumoulin C, Korner-Bitensky N, Tannenbaum C. Urinary incontinence after stroke: does rehabilitation make a difference? A systematic review of the effectiveness of behavioral therapy. Top Stroke Rehabil 2005;12:66-76.

14. Gross JC. A comparison of the characteristics of incontinent and continent stroke patients in a rehabilitation program. Rehabil Nurs 1998;23:132-40.

15. Hu TW, Wagner TH, Bentkover JD, et al. Costs of urinary incontinence and overactive bladder in the United States: a comparative study. Urology 2004;63:461-5.

16. Levy R, Muller N. Urinary incontinence: economic burden and new choices in pharmaceutical treatment. Adv Ther 2006;23:556-73.

17. Wyman JF, Burgio KL, Newman DK. Practical aspects of lifestyle modifications and behavioural interventions in the treatment of overactive bladder and urgency urinary incontinence. Int J Clin Pract 2009;63:1177-91.

18. Godec CJ. "Timed voiding"- a useful tool in the treatment of urinary incontinence. Urology 1984;23:97-100.

18. Newman DK. Conservative therapy for incontinence. In: Goldman HB, Vasavada SP. eds. Female Urology: a Practical Clinical Guide. New York: Humana Press, 2007:63-79.

20. Beetz R. Mild dehydration: a risk factor of urinary tract infection? Eur J Clin Nutr 2003;57:S52-8.

21. Newman DK. Lifestyle interventions. In: Bourcier AP, McGuire EJ, Abrams P, eds. Pelvic floor disorders. Philadelphia: Elsevier Saunders, 2004:269-76.

22. Koskimaki J, Hakama M, Huhtala $\mathrm{H}$, et al. Association of smoking with lower urinary tract symptoms. J Urol 1998;159:1580-2.

23. Kinnunen $\mathrm{O}$. Study of constipation in a geriatric hospital, day hospital, old people's home and at home. Aging (Milano) 1991;3:161-70.

24. Richter HE, Creasman JM, Myers DL, et al. Urodynamic characterization of obese women with urinary incontinence undergoing a weight loss program: the Program to Reduce Incontinence by Diet and Exercise (PRIDE) trial. Int Urogynecol $J$ Pelvic Floor Dysfunct 2008;19:1653-8.

25. Milne JL. Behavioral therapies for overactive bladder: making sense of the evidence. J Wound Ostomy Continence Nurs 2008;35:93-101, 102-103.

26. Burgio KL. Behavioral treatment options for urinary incontinence. Gastroenterology 2004;126:S82-9.

27. Burgio KL. Current perspectives on management of urgency using bladder and behavioral training. J Am Acad Nurse Pract 2004;16:4-7.

28. Burgio KL, Goode PS, Locher JL, et al. Behavioral training with and without biofeedback in the treatment of urge incontinence in older women: a randomized controlled trial. JAMA 2002;288:2293-9.

29. Burgio KL, Locher JL, Goode PS, et al. Behavioral vs drug treatment for urge urinary incontinence in older women: a randomized controlled trial. JAMA 1998;280:1995-2000. 
30. Wyman JF, Fantl JA, Mcclish DK, et al. Comparative efficacy of behavioral interventions in the management of female urinary incontinence. Continence Program for Women Research Group. Am J Obstet Gynecol 1998;179:999-1007.

31. Mattiasson A, Blaakaer J, Hoye K, et al. Simplified bladder training augments the effectiveness of tolterodine in patients with an overactive bladder. BJU Int 2003:91:54-60.

32. Brown $\mathrm{JH}$, Laiken N. Muscarinic receptor agonists and antagonists. In: Brunton L, Chabner B, Knollman B, eds. Goodman \&Gilman's The Pharmacological Basis of Therapeutics. 12th edn. New York: McGraw-Hill, 2011:219-37.

33. Abrams $\mathrm{P}$, Andersson KE. Muscarinic receptor antagonists for overactive bladder. BJU Int 2007;100:987-1006.

34. Lam S, Hilas O. Pharmacologic management of overactive bladder. Clin Interv Aging 2007:2:337-45.

35. Novara G, Galfano A, Secco S, et al. A systematic review and meta-analysis of randomized controlled trials with antimuscarinic drugs for overactive bladder. Eur Urol 2008;54:740-63.

36. Chapple CR. Muscarinic receptor antagonists in the treatment of overactive bladder. Urology 2000;55:33-46, 50.

37. Chapple C, Khullar V, Gabriel Z, et al. The effects of antimuscarinic treatments in overactive bladder: a systematic review and meta-analysis. Eur Urol 2005;48:5-26.

38. Chapple CR, Khullar V, Gabriel Z, et al. The effects of antimuscarinic treatments in overactive bladder: an update of a systematic review and meta-analysis. Eur Urol 2008;54:543-62.

39. George A. Management of urinary incontinence. $P \& T$ 2012;6:345-61.

40. Thomas LH, Cross S, Barrett J, et al. Treatment of urinary incontinence after stroke in adults. Cochrane Database Syst Rev 2008:D4462.

41. Thomas LH, Barrett J, Cross S, et al. Prevention and treatment of urinary incontinence after stroke in adults. Cochrane Database Syst Rev 2005;3:D4462.

42. Dong $\mathrm{H}$, Zhang $\mathrm{X}$. An overview of traditional Chinese medicine. Traditional Medicine in Asia. In: Chaudhary RR, Rafei UM. New Delhi: World Health Organisation. Regional Office South -East Asia, 2002:17-30

43. Yang MX, Zhao L, Yang J, et al. Bibliometric analysis of diseases spectrum of moxibustion therapy. Zhen Ci Yan Jiu 2014;39:247-51.

44. Deng $\mathrm{H}$, Shen $\mathrm{X}$. The mechanism of moxibustion: ancient theory and modern research. Evid Based Complement Alternat Med 2013;2013:379291.

45. Liu HL, Wang LP. [Randomized controlled study on ginger-salt-partitioned moxibustion at shenque (CV 8) on urination disorders poststroke]. Zhongguo Zhen Jiu 2006;26:621-4.

46. Sa J. Sample size of 12 per group rule of thumb for a pilot study. Pharmaceut Statist 2005:287-91.
47. Cheng CW, Fu SF, Zhou QH, et al. Extending the CONSORT statement to moxibustion. J Integr Med 2013;11:54-63.

48. Schick $\mathrm{E}$, Jolivet-Tremblay $\mathrm{M}$, Dupont $\mathrm{C}$, et al. Frequency-volume chart: the minimum number of days required to obtain reliable results. Neurourol Urodyn 2003;22:92-6.

49. Lucas MG, Bosch RJ, Burkhard FC, et al. EAU guidelines on assessment and nonsurgical management of urinary incontinence. Eur Urol 2012;62:1130-42.

50. $\mathrm{Ku} \mathrm{JH}$, Jeong IG, Lim DJ, et al. Voiding diary for the evaluation of urinary incontinence and lower urinary tract symptoms: prospective assessment of patient compliance and burden. Neurourol Urodyn 2004;23:331-5.

51. Wagner TH, Patrick DL, Bavendam TG, et al. Quality of life of persons with urinary incontinence: development of a new measure. Urology 1996;47:67-71, 71-72.

52. Duffy L, Gajree S, Langhorne $P$, et al. Reliability (inter-rater agreement) of the Barthel Index for assessment of stroke survivors: systematic review and meta-analysis. Stroke 2013;44:462-8.

53. Park JE, Lee SS, Lee MS, et al. Adverse events of moxibustion: a systematic review. Complement Ther Med 2010;18:215-23.

54. Ernst E. Testing traditional cupping therapy. J Pain 2009;10:555

55. Yap TL, Cromwell DC, Emberton M. A systematic review of the reliability of frequency-volume charts in urological research and its implications for the optimum chart duration. BJU Int 2007;99:9-16.

56. Brown JS, McNaughton KS, Wyman JF, et al. Measurement characteristics of a voiding diary for use by men and women with overactive bladder. Urology 2003;61:802-9.

57. Jimenez-Cidre MA, Lopez-Fando L, Esteban-Fuertes M, et al. The 3-day bladder diary is a feasible, reliable and valid tool to evaluate the lower urinary tract symptoms in women. Neurourol Urodyn 2013;22:1-5.

58. Bright E, Cotterill N, Drake M, et al. Developing and validating the international consultation on incontinence questionnaire Bladder Diary. Eur Urol 2014:66:294-300.

59. Groutz A, Blaivas JG, Chaikin DC, et al. Noninvasive outcome measures of urinary incontinence and lower urinary tract symptoms: a multicenter study of micturition diary and pad tests. J Urol 2000;164:698-701.

60. Tincello DG, Williams KS, Joshi M, et al. Urinary diaries: a comparison of data collected for three days versus seven days. Obstet Gynecol 2007;109:277-80.

61. Avery KN, Bosch JL, Gotoh M, et al. Questionnaires to assess urinary and anal incontinence: review and recommendations. J Urol 2007; 177:39-49.

62. Linde $\mathrm{K}$, Dincer $\mathrm{F}$. How informed is consent in sham-controlled trials of acupuncture? J Altern Complement Med 2004;10:379-85.

63. Shi GX, Han LL, Liu LY, et al. Acupuncture at local and distant points for tinnitus: study protocol for a randomized controlled trial. Trials 2012;13:224. 